\title{
Riscos da Radiação X e a Importância da Proteção Radiológica na Cardiologia Intervencionista: Uma Revisão Sistemática
}

\author{
Fernando Leyton ${ }^{1}$, Lucia Canevaro ${ }^{2}$, Adriano Dourado 3 , Helio Castello ${ }^{4}$, Alexandre Bacelar ${ }^{5}$, \\ Marcus Teixeira Navarro ${ }^{6}$, Eliseo Vañó ${ }^{7}$, Maria do Socorro Nogueira ${ }^{8}$, Wilson Otto Batista ${ }^{6}$, \\ Tânia A. C. Furquim ${ }^{9}$, Rochelle Lykawka ${ }^{5}$, Camila S. Melo ${ }^{9}$, Flavia Borges ${ }^{8}$, Barbara Rodrigues ${ }^{10}$
}

\section{RESUMO}

Discutimos aqui aspectos vinculados ao enquadramento legal, a recomendações internacionais e a programas de formação em proteção radiológica; ao angiógrafo e à qualidade da imagem; aos efeitos biológicos e aos riscos das radiações ionizantes; às lesões em operadores e pacientes; aos níveis de referência do paciente; ao limite de dose ocupacional e a suas medidas de prevenção. O uso das radiações ionizantes acarreta riscos, que, contudo, justificam-se em procedimentos diagnósticos e terapêuticos. A consciência e o conhecimento desses riscos minimizam o dano, otimizando a qualidade da imagens e o uso seguro das radiações ionizantes. Tem-se demonstrado a ocorrência de cataratas radioinduzidas em trabalhadores de laboratórios de cateterismo. Diversos estudos sugerem que pode haver um risco significativo de opacidade do cristalino, caso não se utilizem adequadamente os dispositivos de proteção radiológica. Adicionalmente, esses tipos de procedimentos intervencionistas são realizados na América Latina, geralmente por médicos especialistas, com a colaboração de enfermeiros, tecnólogos e técnicos, que, muitas vezes, não têm formação adequada em proteção radiológica.

DESCRITORES: Diagnóstico por imagem. Proteção radiológica. Radiação ionizante. Lesões por radiação. Revisão.

\section{ABSTRACT}

\section{Radiation Risks and the Importance of Radiological Protection in Interventional Cardiology: A Systematic Review}

We discuss some aspects related to the legal framework, international recommendations and training programs on radiological protection; image quality and equipment; the biological effects and risks of ionizing radiation; lesions in patients and operators; patient's reference levels; occupational dose limit and preventive actions. The use of ionizing radiation involves risks that are justified in diagnostic and therapeutic procedures. The awareness and knowledge of these risks minimizes the damage, optimizing the quality of images and safe use of ionizing radiation. There is evidence of radiationinduced cataracts in individuals who work in catheterization laboratories. Several studies suggest there may be a significant risk of lens opacity, if radiological protection devices are not properly used. Additionally, these interventional procedures are performed in Latin America, usually by medical specialists in collaboration with nurses, technologists and technicians, who often do not have adequate training in radiological protection.

DESCRIPTORS: Diagnostic imaging. Radiation protection. Radiation, ionizing. Radiation injuries. Review.

\footnotetext{
Faculdade de Saúde e Odontologia, Universidade Diego Portales, Santiago, Chile.

2 Instituto de Radioproteção e Dosimetria, Comissão Nacional de Energia Nuclear, Rio de Janeiro, RJ, Brasil.

3 Hospital Santa Isabel, Salvador, BA, Brasil.

${ }^{4}$ Hospital Bandeirantes, São Paulo, SP, Brasil.

${ }^{5}$ Serviço de Física Médica e Radioproteção, Hospital de Clínicas de Porto Alegre, Universidade Federal do Rio Grande do Sul, Porto Alegre, RS, Brasil.

${ }^{6}$ Laboratório de Física Radiológica, Instituto Federal da Bahia, Salvador, BA, Brasil.

${ }^{7}$ Departamento de Radiologia, Universidade Complutense de Madrid e Hospital São Carlos, Madri, Espanha.

${ }^{8}$ Centro de Desenvolvimento da Tecnologia Nuclear, Belo Horizonte, MG, Brasil.

${ }^{9}$ Instituto de Física, Universidade de São Paulo, São Paulo, SP, Brasil.

${ }^{10}$ Secretaria de Saúde do Distrito Federal, Distrito Federal, Brasil.
}

\begin{abstract}
Os autores deste manuscrito participam do projeto nacional BRA 9056 "National Assessment of Quality Control and Radiation Protection in Interventional Cardiology Departments" da International Atomic Energy Agency (IAEA). O projeto é uma parceria entre o Instituto de Radioproteção e Dosimetria (IRD) da Comissão Nacional de Energia Nuclear (CNEN); a Sociedade Brasileira de Hemodinâmica e Cardiologia Intervencionista $(\mathrm{SBHCl})$; o Centro de Desenvolvimento da Tecnologia Nuclear (CDTN); o Instituto de Física da Universidade de São Paulo (IF-USP); o Laboratório de Física Radiológica do Instituto Federal da Bahia (IFBA); o Hospital das Clínicas de Porto Alegre da Universidade Federal do Rio Grande do Sul (UFRGS); e a Secretaria de Saúde do Distrito Federal. O projeto se propõe a identificar o parque tecnológico atual em Cardiologia Intervencionista no Brasil, caracterizar os angiógrafos e fazer um levantamento das doses ministradas a pacientes e profissionais durante os procedimentos.
\end{abstract}

Correspondência: Fernando Leyton. Centro de Desenvolvimento da Tecnologia Nuclear - Avenida Presidente Antônio Carlos, 6.627 Campus da UFMG - CEP: 31270-901 - Belo Horizonte, MG, Brasil E-mail: leyton.fernando@gmail.com

Recebido em: 19/9/2013 • Aceito em: 12/2/2014 
A plicações médicas representam a principal fonte de exposição artificial da população mundial às radiações ionizantes. O National Council on Radiation Protection and Measurements (NCRP), ${ }^{1}$ nos Estados Unidos, estima que a exposição à radiação para a produção de imagens médicas aumentou em $600 \%$ entre 1980 e 2006. De acordo com o United Nations Scientific Committee on the Effects of Atomic Radiation (UNSCEAR), ${ }^{2}$ os procedimentos de cardiologia intervencionista representam a terceira maior contribuição às doses coletivas (uma dose coletiva caracteriza o impacto radiológico de um procedimento ou fonte ionizante), depois da tomografia computadorizada e da medicina nuclear.

A introdução de novas tecnologias de imagem no campo diagnóstico e terapêutico e a permanente modernização dos aparelhos de raios $X$ ampliaram $O$ espectro de atuação da cardiologia intervencionista, responsável, atualmente, por inúmeros procedimentos em doenças coronária, valvar e congênita. Para garantir o bom funcionamento dos equipamentos e gerenciar adequadamente os riscos radiológicos, devem ser estabelecidos programas de garantia e de controle de qualidade nos serviços de Cardiologia Intervencionista, conforme recomendação da International Atomic Energy Agency (IAEA).

Devido aos elevados níveis de exposições que podem ser atingidos em alguns procedimentos da Cardiologia Intervencionista, é possível observar nos pacientes efeitos secundários a doses elevadas (determinísticos) ou proporcionais à dose recebida, sem limiar (estocásticos). Por outro lado, pelo fato de o procedimento requerer a presença do médico ao lado do paciente, também é possível que ocorram efeitos determinísticos nesses profissionais, como epilação e catarata.

\section{Recomendações internacionais e marco legal em proteção radiológica}

Ao contrário do que acontece na Europa e nos Estados Unidos, ${ }^{3-7}$ na América Latina, em geral, não há enquadramento legal o suficiente para regular o uso seguro das radiações ionizantes na Medicina. A consciência de que existem procedimentos intervencionistas que podem implicar em altas doses de radiação ao paciente e ao operador tem motivado as organizações internacionais a publicarem recomendações que ajudem a conjugar as necessidades da Cardiologia Intervencionista aos altos padrões de segurança. Assim, a Diretiva Europeia 97/43/ Euratom ${ }^{5}$ sobre exposições médicas determina requisitos para a prática da Cardiologia Intervencionista. Guia publicado pela Comissão Europeia, visando à educação e ao treinamento em proteção radiológica para as exposições médicas, também contém recomendações acerca de programas de formação e acreditação. . $^{6-8}$

Já o documento "Radiation Safety in the Practice of Cardiology", ${ }^{4}$ publicado em conjunto pelo American

College of Cardiology (ACC), pela North American Society of Pacing and Electrophysiology (NASPE), pela Society for Cardiac Angiography and Interventions (SCAI) e pela American Society of Nuclear Cardiology (ASNC), faz recomendações sobre proteção radiológica, para que os procedimentos da prática cardiológica sejam realizados com níveis de segurança adequados. Da mesma forma, a publicação "Normas de Seguridad del OIEA № GSR Part 3"9 e as publicações 60, 103 e 105, da International Commission on Radiological Protection (ICRP), ${ }^{10-12}$ estabelecem os critérios básicos para radioproteção, estabelecendo princípios de justificação, otimização e limitação das doses para os indivíduos ocupacionalmente expostos (IOE), além dos valores de referência de dose para as práticas médicas.

Chambers et al., ${ }^{13,14}$ que faziam parte da SCAI, publicaram artigo cujo enfoque prático servia à assistência aos serviços de Cardiologia Intervencionista no estabelecimento de programas de segurança e proteção radiológica. Nele, foram revisados: a terminologia básica para o planejamento da dose pré-procedimento; o gerenciamento da dose em tempo real; a documentação da dose de radiação nos registros; a notificação de dose alta para o paciente e o médico de referência; os elementos de proteção e equipamento de imagens; e o treinamento. $\mathrm{O}$ artigo ainda estabeleceu que os médicos e equipe médica envolvida no cateterismo deveriam ser treinados nos princípios básicos da física das radiações, segurança e proteção radiológica. Um físico médico deve participar, junto da equipe médica, da seleção do equipamento, da educação do pessoal e da medição da dose. Uma melhor qualidade da imagem, com exposição otimizada à radiação, assegura o melhor cuidado ao paciente, bem como o aprimoramento da prática.

Na publicação 85 do ICRP, ${ }^{15}$ recomenda-se a utilização de três dosímetros para os indivíduos mais expostos. Sugere-se colocar um dosímetro pessoal principal sob o avental plumbífero à altura do tórax, direcionado para a fonte de radiação; um segundo dosímetro localizado acima do avental, na altura do pescoço; e um terceiro perto do olho (cristalino) ou na região da mão (Figura 1 ). A normativa brasileira estabelece o uso de um dosímetro na altura do tórax por fora do avental plumbífero. ${ }^{16}$

A IAEA recomenda incluir na legislação de proteção radiológica a implantação dos programas de garantia e de controle de qualidade, com os testes de aceitação e o "comissionamento" ou caracterização dos sistemas que produzem radiações ionizantes usadas em procedimentos intervencionistas. No Brasil, a Agência Nacional de Vigilância Sanitária (ANVISA) do Ministério da Saúde publicou, em 1998, a Portaria SVS/MS 453/98, ${ }^{16}$ que estabelece requisitos de proteção radiológica e controle de qualidade para a radiologia médica, odontológica e intervencionista.

Embora ainda não exista um regulamento específico para o uso das radiações ionizantes em Cardiologia 


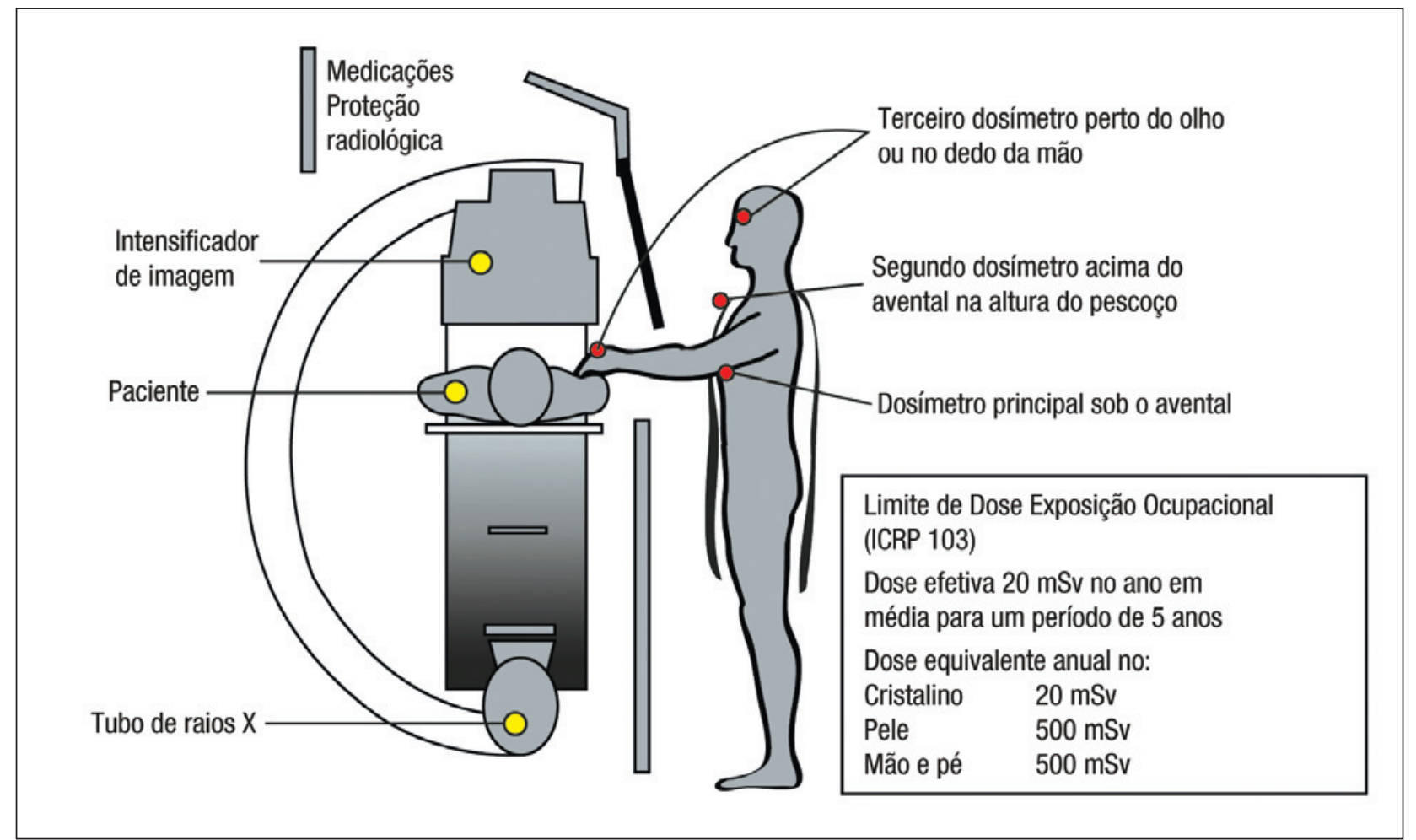

Figura 1. Posição dos dosímetros para descrever a exposição do staff em procedimentos intervencionistas. O dosímetro pessoal principal tem que estar sob o avental plumbífero, à altura do tórax, direcionado a fonte de radiação. O segundo dosímetro pode ser localizado acima do avental, na altura do pescoço, e o terceiro perto do olho ou mão.

Intervencionista, a Norma Regulamentadora - Segurança e Saúde no Trabalho em Estabelecimentos de Saúde, conhecida como NR-32, ${ }^{17}$ estabelece as diretrizes básicas para a proteção à saúde dos trabalhadores dos serviços de saúde. Nela, fica expresso que os equipamentos de fluoroscopia devem possuir "cortina ou saiote plumbífero inferior e lateral para proteção do operador contra radiação espalhada". Na Europa e em outros países, ${ }^{5-7}$ não é permitido comercializar um equipamento de angiografia sem os elementos externos de proteção radiológica e nem sem o medidor do produto kerma-área (ou produto dose-área $-\mathrm{P}_{K A}$ ), que indica a dose de radiação que o paciente recebe $\mathrm{e}$ constitui ferramenta indispensável para a segurança e a otimização dos procedimentos intervencionistas. No Brasil, as câmaras para medir o $P_{K A}$ e os elementos externos de proteção radiológica, como o saiote e o biombo de teto, ainda não são habituais, mas deveriam ser considerados partes indispensáveis na aquisição de um equipamento angiográfico.

\section{Formação em proteção radiológica}

Na América Latina, os procedimentos intervencionistas são realizados por médicos especialistas acompanhados por enfermeiros, tecnólogos médicos e técnicos, que, muitas vezes, não têm formação adequada em proteção radiológica. Pesquisa realizada por Vano et al. ${ }^{18}$ em dez centros de Cardiologia Intervencionista de países latino-americanos - o Brasil inclusive - afirmou que apenas $64 \%$ dos IOE utilizavam o monitor individual (dosímetro) e apenas 36\% deles reconheceram saber o que significava sua dosimetria. Sobre o conhecimento e o uso do angiógrafo, apenas $14 \%$ demonstraram conhecer adequadamente o equipamento e apenas $27 \%$ tinham conhecimento dos resultados do controle de qualidade. Em outra pesquisa realizada pela IAEA, da qual participaram cardiologistas de mais de 56 países, os resultados mostraram que entre 33 e $77 \%$ dos cardiologistas intervencionistas usavam regularmente seus dosímetros. ${ }^{19}$

Publicação conjunta do ACC, da American Heart Association e do American College of Physicians (ACC) $\mathrm{AHA} / \mathrm{ACP})^{20}$ estabeleceu, em 2005, a competência clínica no conhecimento médico, para aprimorar a segurança do paciente, bem como a qualidade de imagem. Outras publicações do ACC, principalmente as que tratavam da realização de procedimentos de forma segura, levaram à publicação do documento "Cardiac Catheterization Laboratory Standards: a Report of the American College of Cardiology Task Force on Clinical Expert Consensus Documents", ${ }^{21}$ que estabeleceu os padrões técnicos para os laboratórios de cateterismo cardíaco, tendo um de seus capítulos dedicado à proteção radiológica. 
O regulamento das radiações ionizantes nas exposições médicas da Comissão Europeia ${ }^{6}$ e do Reino Unido (o Ionising Radiation (Medical Exposure) Regulation - IRMER) ${ }^{7}$ estabeleceu o adequado treinamento, tanto teórico como prático, que um operador de procedimentos intervencionistas deve receber. Atualmente, na Espanha, os cardiologistas intervencionistas devem dispor de acreditação em proteção radiológica, especialmente focada na área intervencionista. ${ }^{22}$

\section{O angiógrafo e a qualidade da imagem}

Médicos intervencionistas e físicos médicos deveriam participar do processo de compra e de configuração de um equipo de angiografia. Devem ser determinados quais procedimentos serão realizados para que seja escolhido o angiógrafo mais apropriado e que esteja de acordo com a demanda. A ampla variedade de procedimentos que podem ser realizados e as grandes diferenças entre os componentes do hardware, os softwares e configurações necessárias para diferentes aplicações fazem com que o angiógrafo seja um equipamento de alta complexidade.

Para realizar os procedimentos em Cardiologia Intervencionista, é necessário um angiógrafo especialmente desenhado e configurado para tal prática médica. $\mathrm{O}$ equipamento deve ser instalado em uma sala que permita o livre movimento do arco C, a circulação do staff e a instalação de todos os dispositivos necessários para a prática intervencionista, como, por exemplo, os monitores, o biombo de teto, o desfibrilador, as bombas, os injetores, a máquina de anestesia e os monitores fisiológicos, entre outros. A sala deve assegurar todos os cuidados ao paciente e minimizar os riscos para o paciente e a equipe clínica.

As características desejáveis para um angiógrafo utilizado em cardiologia intervencionista são:

1. geometria: o tubo de raios $X$ e o receptor da imagem giram em torno de um ponto chamado "isocentro", no qual deve ser posicionada a região anatômica de interesse no estudo;

2. modos de fluorosocopia: a escolha de diferentes taxas de pulso tem que estar disponível para o operador. A incorporação da fluoroscopia pulsada foi um importante avanço na redução da dose ao paciente;

3. filtros: diferentes espessuras de filtros estão disponíveis, em função da espessura e da densidade tecidual do paciente. Os filtros auxiliam na qualidade da imagem e no controle dosimétrico do paciente;

4. ajuste de colimação: a colimação virtual apresenta a posição das lâminas do colimador sobre a imagem, enquanto as lâminas são ajustadas. Esse recurso elimina a irradiação do paciente durante o ajuste do colimador. A colimação adequada diminui a dose do paciente e do operador e melhora a qualidade da imagem;
5. programação anatômica: permite o controle do gerenciamento da dose e da qualidade da imagem, selecionando a região do corpo e o tamanho do paciente que será irradiado;

6. aquisição digital: o processamento de imagens não digitais é muito limitado, em comparação com o das imagens digitais. O formato para a edição de imagens digitais e comunicação em medicina (DICOM) representa o principal tipo de arquivo utilizado para a aquisição, manipulação e gerenciamento das imagens em Cardiologia Intervencionista. O cabeçalho DICOM fornece importantes informações a respeito dos protocolos adotados no centro para cada procedimento;

7. armazenamento da fluoroscopia: essa característica mostra a última imagem fluoroscópica e permite o armazenamento retrospectivo dos últimos segundos de fluoroscopia. O recurso permite ao operador armazenar até 300 imagens da sequência de fluoroscopia e admite o registro da anatomia cardíaca sem a necessidade de irradiar o paciente novamente;

8. monitores de radiação: permite a visualização em tempo real do kerma no ar (Ka) e do $\mathrm{P}_{K A}$. Esse monitor possibilita a avaliação do risco de radiação do paciente durante o procedimento. O serviço de Cardiologia Intervencionista deve registrar as doses dos pacientes e implantar um sistema de seguimento que superasse a dose de alerta;

9. blindagem/elementos de proteção radiológica externos: esses escudos consistem em cortinas de chumbo montadas no trilho da mesa do paciente (saiote) e em outro escudo montado com um braço articulado, colado ao teto da sala de procedimento (o biombo de teto). A espessura típica equivalente é de $0,5 \mathrm{mmPb}$. O biombo de teto tem uma vantagem se comparado aos óculos de proteção, pois, além de proteger o cristalino, protege também a cabeça, a tiroide, os braços e toda a porção superior do tronco do operador;

10. alertas audíveis: um alerta sonoro deve ser ativado na sala de procedimento sempre que os raios $X$ são produzidos. Sons diferentes para diferentes modos de operação são desejáveis.

Um angiógrafo para exames complexos e que não possua a maioria das características mencionadas não deve ser utilizado na Cardiologia Invasiva. ${ }^{23}$

O ajuste adequado de todos os parâmetros do angiógrafo, tais como a taxa e a largura do pulso, a corrente e a tensão do tubo, a filtração e o tamanho do ponto focal e do campo de visão, contribui para a otimização da exposição do paciente e a melhoria da qualidade da imagem.

Atualmente, os sistemas fluoroscópicos utilizados em procedimentos de Cardiologia Intervencionista utilizam dois tipos de tecnologias para a detecção da imagem: o intensificador de imagem com dispositivo 
de carga acoplada e os detectores flat panel, que chegaram para substituir o intensificador de imagem. Existem numerosos estudos que comparam ambas as tecnologias, destacando-se que ainda não existem diferenças significativas no nível de dose e na qualidade da imagem entre elas. ${ }^{24,25}$ Por outro lado, enfatiza-se também o maior potencial para diminuir as doses e melhorar a qualidade da imagem dos detectores flat panel.

No Brasil, os trabalhos de Medeiros et al. ${ }^{26}$ e Vargas et al. ${ }^{27}$ mostraram que o angiógrafo com flat panel apresentou as maiores taxa de Ka máxima na entrada da pele do paciente, chegando a ser $65 \%$ maior se comparado aos sistemas que utilizam intensificador da imagem. Assim, a redução de dose não é inerente ao sistema flat panel, portanto, é necessária a otimização da dose por um físico médico qualificado.

A qualidade da imagem e a dose estão fortemente relacionadas. A diminuição da dose provoca uma diminuição na qualidade da imagem, de tal modo que o excesso de zelo para se obterem doses baixas pode resultar em angiógrafos gerando uma imagem com baixa qualidade.

Uma imagem de qualidade é fundamental à orientação do procedimento clínico. Assim, a avaliação da qualidade da imagem pode ser realizada de forma objetiva ou subjetiva. ${ }^{28}$ É necessário um phantom específico para sistemas que utilizam fluoroscopia para avaliação da qualidade da imagem. A avaliação subjetiva necessita de um observador que informe o número de grupos de alta resolução visíveis e os objetos de baixo contraste. Esse tipo de avaliação está sujeita à variabilidade do observador. A avaliação objetiva (numérica) pode ser realizada com as imagens DICOM. As avaliações podem incluir a função de transferência modulada, o ruído, o contraste e a relação sinal-ruído. As avaliações numéricas da qualidade da imagem são mais demoradas que as subjetivas, mas muito consistentes.

A verificação da qualidade da imagem e das doses, para os diferentes modos de exposição, constitui a base dos testes de constância (testes de verificação de desempenho) e do comissionamento. Esse processo é decisivo para o aprimoramento da qualidade das imagens clinicamente aceitáveis e obtidas com taxas de dose tão baixas quanto razoavelmente exequíveis. Assim, é indispensável a caracterização completa do sistema de raios $X$, durante o comissionamento e depois de grandes mudanças. Se essa tarefa ainda não foi realizada, esse é o primeiro passo para um processo de otimização.

\section{Efeitos biológicos das radiações ionizantes}

A maioria dos efeitos indesejados decorrentes da exposição às radiações ionizantes podem se agrupar em duas categorias:

1. efeitos estocásticos: aqueles cuja probabilidade de ocorrência é proporcional à dose de radiação re- cebida, sem a existência de limiar. O desenvolvimento de câncer em indivíduos expostos, devido à mutação de células somáticas ou por uma doença hereditária em sua progênie, é exemplo;

2. efeitos determinísticos: efeitos causados por irradiação total ou localizada de um tecido, levando a um grau de morte celular não compensado pela reposição ou pelo reparo, com prejuízos detectáveis no funcionamento do tecido ou órgão. Existe um limiar de dose, abaixo do qual a perda de células é insuficiente para prejudicar o tecido ou órgão de um modo detectável.

A ICRP $103^{11}$ adverte que alguns efeitos associados à radiação, diferentes do câncer, ainda não são entendidos suficientemente para serem colocados em alguma das categorias mencionadas.

Os dois exemplos mais frequentes de reações tissulares (efeito determinísticos) de interesse em Cardiologia Intervencionista são a formação de catarata, em médicos, e lesões na pele, em pacientes. ${ }^{29}$

O estudo dos efeitos determinísticos se baseiam na análise da distribuição espacial e temporal das doses sobre o tecido. Com uma dose única de 6 a 8 Gy, em um campo de $5 \mathrm{~cm}^{2}$, surge um eritema, que aumenta na primeira semana, mas desaparece progressivamente depois de 10 dias. Com o aumento da dose, a reação eritematosa chega a um máximo em 2 semanas e dura de 20 a 30 dias. No caso de doses ainda maiores, podem ocorrer a descamação seca ou úmida e, inclusive, a necrose. ${ }^{30}$

A grandeza apropriada para planejar a exposição de pacientes e as avaliações do risco-beneficio é a dose equivalente ou a dose absorvida nos tecidos irradiados (cuja unidade é o Sievert - Sv e o Gray Gy, respectivamente). A dose efetiva pode ser valiosa para comparar a dose de diferentes procedimentos diagnósticos, além de pôr em paralelo a utilização de tecnologias e de procedimentos similares em diferentes hospitais e países. ${ }^{11}$

As revisões dos dados biológicos e clínicos realizadas pela ICRP 103 têm estimado que, para uma dose absorvida de até 100 mGy, nenhum tecido expressa dano funcional clinicamente relevante. ${ }^{11}$ Essa estimativa se aplica a doses agudas únicas ou a situações em que as dose são recebidas de forma prolongada, como as exposições anuais. No caso do câncer, os estudos mostraram evidências do risco da radiação para doses ao redor de $100 \mathrm{mSv}$ ou possivelmente menores.

No caso de doenças hereditárias, ainda não existe evidência direta de que a exposição à radiação dos pais leve a maior prevalência de doenças hereditárias nos filhos, embora o ICRP 103 tenha considerado que a radiação cause efeito hereditário em animais de experimentação. Essas observações experimentais foram suficientes para que os riscos para as futuras 
gerações fossem incluídos nos sistemas de proteção. De acordo com a ICRP 103, existe risco de reação de tecido e malformação do embrião e do feto irradiado no útero. No caso do embrião, pode haver efeitos letais no período de pré-implantação. A respeito das malformações, os padrões de radiossensibilidade in utero dependem da idade gestacional. O limiar para a indução de malformações é da ordem de 100 mGy e, abaixo desse valor, não se esperam malformações.

O sistema prático de proteção radiológica recomendada pelo ICRP 103 está baseado na suposição de que, para doses $>100 \mathrm{mSv}$, um aumento na dose produza um incremento diretamente proporcional à probabilidade de desenvolver câncer ou efeitos hereditários decorrentes da radiação. ${ }^{11}$ Esse modelo de resposta à dose é conhecido como "linear sem limiar" (LNT, sigla do inglês linear-non-threshold). Esse critério foi compartilhado por UNSCEAR 2000, ${ }^{31}$ NCRP 2001-20091,32 e NAS/NRC 2006. ${ }^{33}$ A publicação 99 do ICRP $2005^{34}$ considerou que a adoção do modelo LNT, combinado com um valor estimado do fator de eficácia da dose e taxa de dose, proporciona uma base prudente para os propósitos práticos da proteção radiológica, ou seja, a gestão dos riscos da exposição a doses baixas de radiação.

\section{Lesões em operadores e pacientes}

Infelizmente alguns pacientes submetidos a procedimentos intervencionistas têm sofrido danos causados pela radiação (Figuras 2 e 3)..$^{35,36} \mathrm{Um}$ dos primeiros registros de uma necrose na pele por cateterismo guiado por fluoroscopia foi em $1976 .{ }^{37}$ Mas foi só na década de 1990 é que o Food and Drug Administration (FDA) publicou alerta chamando a atenção para os riscos das lesões de pele por radiação em pacientes submetidos a procedimentos médicos com fluoroscopia. ${ }^{3}$

No Chile, com apoio da IAEA, foi realizada uma pesquisa nos laboratório de Cardiologia Intervencionista. Dos entrevistados, 90\% declararam desconhecer casos de lesões secundárias à radiação e $84 \%$ declararam que nunca presenciaram exposição excessiva dos pacientes. ${ }^{38}$ No Brasil, encontramos dificuldades para notificar esses casos.

A ocorrência de cataratas radioinduzidas entre os trabalhadores de laboratórios de radiologia intervencionista tem sido demonstrada (Figura 4). ${ }^{15,39-41}$ Vano et al. ${ }^{42}$ revelaram que $50 \%$ dos médicos cardiologistas expostos a radiações ionizantes apresentaram opacidade do cristalino. Estudos com o objetivo de avaliar retrospctivamente as lesões do cristalino e a dose de radiação, como o Retrospective Evaluation of Lens Injuries and Dose (RELID) da IAEA, ${ }^{43}$ contribuíram para a diminuição do limite da dose para essa região. Pelo fato de os médicos intervencionistas e sua equipe permanecerem várias horas por dia perto da fonte de raios $X$, mesmo em condições normais de trabalho, os níveis de exposição aos olhos ainda podem ser consideravelmente elevados.

O estudo de Russo et al. ${ }^{44}$ mostra que os cardiologistas intervencionistas apresentam aumento do dano

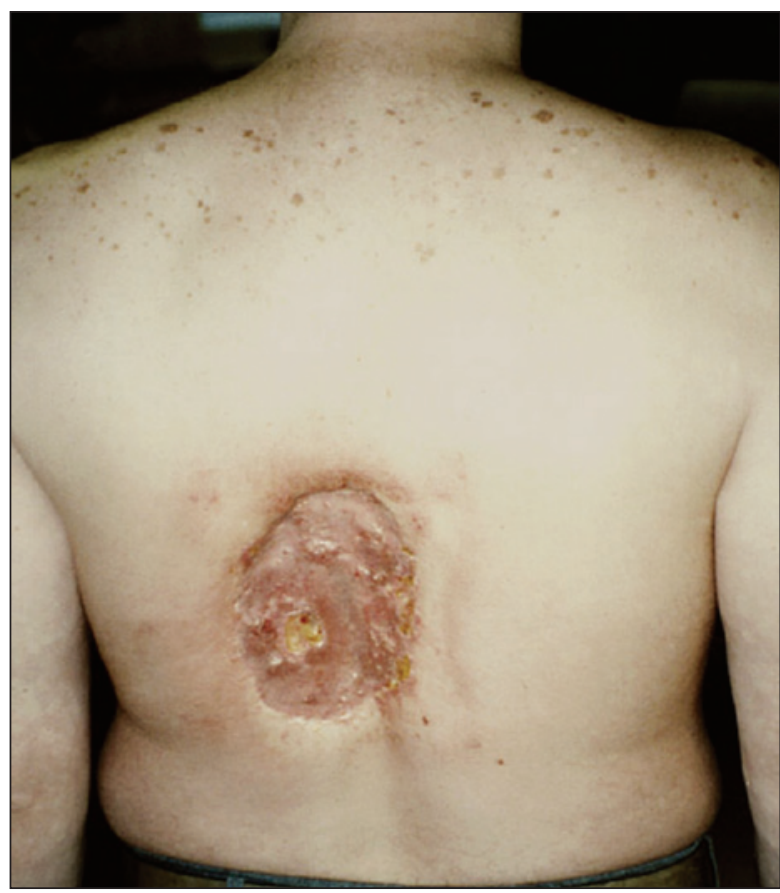

Figura 2. Lesão na pele em homem de 40 anos submetido a múltiplas coronariografias. Aproximadamente 18 a 21 meses depois do procedimento, evidencia-se necrose dos tecidos. Fonte: Shope TB. Radiationinduced skin injuries from fluoroscopy [image]. In: U.S. Food and Drug Administration. Radiation-Imitting Products [Internet]. 2010 [cited 2013 Aug 15]. Available from: http://www.fda.gov/Radiation-EmittingProducts/ RadiationEmittingProductsandProcedures/Medicallmaging/MedicalX-Rays/ ucm116682.htm

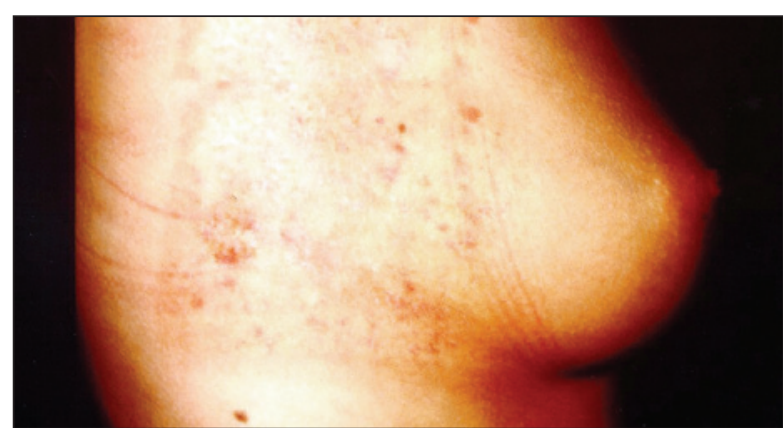

Figura 3. Adolescente de 17 anos, com história de arritmia cardíaca, submetida a duas ablações com 13 meses de diferença. Fotografia tirada 2 anos depois da última intervenção mostrando uma placa endurecida e atrofia no tórax lateral, comprometendo a mama à direita. O endurecimento resultou na limitação do movimento do braço direito. Há aumento do risco de câncer de mama. Fonte: Vañó E, Arranz L, Sastre JM, Moro C, Ledo A, Gárate MT, et al. Dosimetric and radiation protection considerations based on some cases of patient skin injuries in interventional cardiology. Br J Radiol. 1998;71(845):510-6. Com permissão do British Institute of Radiology. 
ao DNA somático, ou seja, uma exposição crônica a baixas doses de radiação ionizante induz a respostas adaptativas, bioquímicas e celulares para baixas doses, mesmo em níveis considerados seguros de exposição, motivo pelo qual a adoção de medidas de proteção radiológicas é fundamental.

Almeida Junior et al. ${ }^{45}$ mostraram que doses anuais do IOE no laboratório de intervenção facilmente excedem o limite legal permitido a trabalhadores expostos à radiação ionizante, caso não sejam utilizados os dispositivos de proteção radiológica durante os procedimentos intervencionistas. ${ }^{46}$

Ocorrências de catarata são consideradas um efeito determinístico. A ICRP observou evidências epidemiológicas e emitiu, em abril de 2011, uma declaração reduzindo o limiar de dose para o qual esse efeito deve acontecer. ${ }^{47}$ De acordo com essa declaração, o limiar de dose absorvida para lesões no cristalino passou a 0,5 Gy (500 mGy) e o limite ocupacional de dose equivalente no cristalino a $20 \mathrm{mSv}$ por ano. Estimativas indicam que as doses acumuladas por médicos intervencionistas e pela equipe médica, durante a vida profissional, podem superar esses valores estabelecidos pelas normas internacionais

\section{Níveis de referência para paciente e limite de dose ocupacional}

Grandes diferenças nas exposições em um mesmo exame diagnóstico ou terapêutico indicam que existe um grande potencial de redução de dose. ${ }^{28} \mathrm{O}$ conceito de níveis de referência foi desenvolvido como ferramenta para a otimização da proteção para a exposição dos pacientes. Os níveis de referência são definidos como valores de grandeza medida, acima dos quais alguma

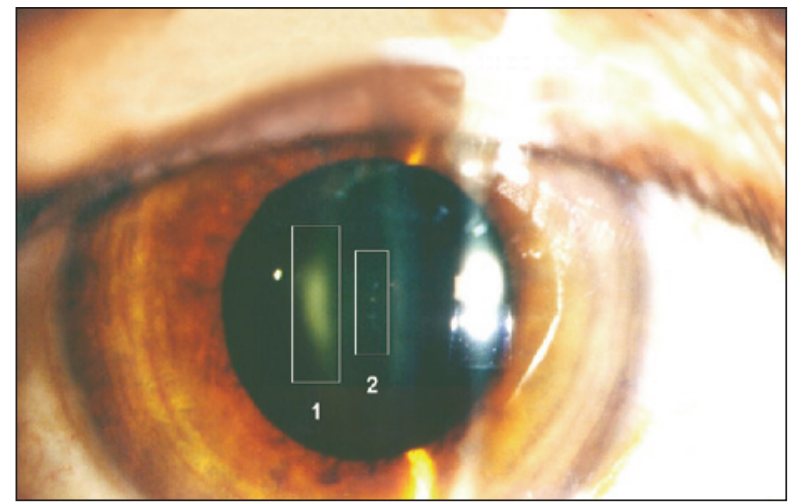

Figura 4. Opacidade no cristalino radioinduzida em um especialista em Radiologia Intervencionista submetido a altos níveis de radiação espalhada, utilizando um angiógrafo não otimizado com tubo de raios $\mathrm{X}$ acima da mesa. $\mathrm{O}$ número 1 indica opacidade subcapsular posterior; o número 2, opacidades puntiformes perinucleares. Fonte: Vañó E, González L, Beneytez F, Moreno F. Lens Injuries induced by ocupational exposure in non-optimized interventional radiology laboratories. Br J Radiol. 1998;71(847):728-33. Com permissão do British Institute of Radiology. ação ou decisão específica deve ser tomada. São usados para identificar situações nas quais as doses ao paciente estão frequentemente fora do usual. Se for constatado que as exposições durante os procedimentos são consistentemente diferentes do nível de referência, os procedimentos e os equipamentos devem ser revistos, de modo a determinar se estes foram adequadamente ajustados. Os níveis de referência estão destinados para uso nos programas de garantia da qualidade, servindo como guias para determinar a revisão detalhada do equipamento e também para garantir o melhor rendimento do operador.

Nos países que têm implantado os níveis de referência, os médicos e a equipe médica dispõem de uma ferramenta de fácil aplicação para comparar as doses de radiação entregue aos pacientes. Isso tem produzido maior consciência dos profissionais para as doses de radiação associadas às práticas, estimulando as ações corretivas em cada instituição. ${ }^{11,28,48}$ Nesses países, tem se observado a redução significativa da dose de radiação ao paciente. ${ }^{18,49}$ Os níveis de referência, portanto, estão bem estabelecidos para os procedimentos comuns e são exigidos pelas recomendações internacionais. ${ }^{9,11,28,48}$

As doses nos procedimentos de Cardiologia Intervencionista podem chegar a variar em um fator 10 (Tabela 1) em procedimentos complexos, como a intervenção em uma oclusão coronária crônica, ${ }^{50}$ a substituição de uma válvula aórtica transtorácica, ${ }^{51}$ ou o reparo de um aneurisma endovascular torácico-abdominal, ${ }^{52}$ os quais podem exceder a dose efetiva de $100 \mathrm{mSv}$.

$\mathrm{O}$ produto kerma área $\left(\mathrm{P}_{\mathrm{KA}}\right)$ é uma grandeza que representa a energia da radiação transmitida ao paciente durante o procedimento, sendo expressa em Gy. $\mathrm{cm}^{2}$. Essa grandeza é calculada pelo sistema de angiografia e apresentada no monitor da sala durante o procedimento. Conhecendo o $\mathrm{P}_{\mathrm{KA}}$ do paciente, é possível estimar a dose que o operador recebe. A IAEA, na publicação Safety Report Series $n^{\circ}$. 59, ${ }^{28}$ estabeleceu níveis de referência de $50 \mathrm{~Gy} . \mathrm{cm}^{2}$ para a angiografia coronária diagnóstica e de 125 Gy. $\mathrm{cm}^{2}$ para a intervenção coronária percutânea (ICP), sendo considerados o tempo de fluoroscopia, o número de imagens e a complexidade do procedimento.

Na literatura nacional, têm sido pouco relatadas as doses em pacientes da Cardiologia Intervencionista. Pesquisas realizadas no Estado do Rio Grande do Sul26,27,55 mostraram que o $\mathrm{P}_{\mathrm{KA}^{\prime}}$ em procedimentos de Cardiologia Intervencionista, variou entre 41 a 55 Gy.cm² e 62 a $103 \mathrm{~Gy} . \mathrm{cm}^{2}$, para os procedimentos diagnósticos e terapêuticos, respectivamente (valores do terceiro quartil).

O estudo de Azevedo et al. ${ }^{55}$ determinou o impacto das lesões complexas na exposição radiológica durante ICP. Foi concluído que a exposição radiológica é significativamente maior nos pacientes submetidos a ICP em lesões complexas do tipo B2/C, comparadas às 
TABELA 1

Dose efetiva para exames comuns em Cardiologia ${ }^{29}$ associada ao número equivalente de radiografia de tórax e ao período aproximado de radiação natural que um indivíduo recebe em 1 ano

\begin{tabular}{|c|c|c|c|}
\hline Procedimento & $\begin{array}{c}\text { Dose efetiva } \\
(\mathrm{mSv})\end{array}$ & $\begin{array}{l}\text { Número de radiografias de tórax } \\
\text { posteroanterior (para 0,02 mSv) }\end{array}$ & $\begin{array}{l}\text { Período aproximado de } \\
\text { radiação natural (anos) }\end{array}$ \\
\hline Radiografia de tórax posteroanterior ${ }^{53}$ & 0,02 & 1 & 0,008 \\
\hline \multicolumn{4}{|l|}{ Intervenções } \\
\hline Angiografia da artéria pulmonar ou aorta ${ }^{54}$ & $5(4-9)$ & 250 & 2,1 \\
\hline Angiografia de cabeça e/ou pescoço $0^{54}$ & $5(1-20)$ & 250 & 2,1 \\
\hline Angiografia de coronária diagnóstica ${ }^{53}$ & $7(2-16)$ & $350(100-800)$ & 2,9 \\
\hline Intervenção coronária percutânea ${ }^{53}$ & $15(7-57)$ & $750(350-2.800)$ & 6,3 \\
\hline Ablação cardíaca por radiofrequência ${ }^{53}$ & $15(7-57)$ & $750(350-2.800)$ & 6,3 \\
\hline Dilatação de uma oclusão coronária crônica ${ }^{50}$ & $81(17-194)$ & $4.050(850-9.600)$ & 33,7 \\
\hline \multicolumn{4}{|l|}{ Tomografia computadorizada } \\
\hline Angiografia coronária por tomografia computada, $64-$ slice $^{53}$ & $15(3-32)$ & $750(150-1.600)$ & 6,3 \\
\hline
\end{tabular}

do tipo A/B1, e que o peso do paciente e o tipo de lesão (B2/C) são preditores de exposição radiológica aumentada. Também relataram que $14 \%$ dos pacientes submetidos a ICP ultrapassaram o nível de dose de 2 Gy, que, potencialmente, pode produzir lesão no paciente.

Vargas et al. ${ }^{27}$ também relataram que a dose de alguns pacientes ultrapassou o nível de referência de 2 Gy. O estudo mostrou que a exposição radiológica foi $>2$ Gy em 3,6\% dos procedimentos do grupo com menos de $79 \mathrm{~kg}$, em 16,3\% do grupo entre 80 e $99 \mathrm{~kg}$, e em $10,7 \%$ do grupo com mais de $100 \mathrm{~kg}$. Dessa maneira, o peso do paciente exerce impacto significativo na exposição radiológica.

Por outro lado, Mattos et al. ${ }^{56}$ demostraram que pacientes submetidos a procedimentos cardiológicos invasivos, tanto diagnósticos como terapêuticos, eram expostos a maiores níveis de radiação pela via de acesso radial, e sugeriram que a experiência do operador pode reduzir, de forma significativa, o nível de dose do procedimento.

Esses estudos evidenciaram o grande potencial de otimização da dose dos procedimentos intervencionistas. As recomendações internacionais estabelecem que, no caso de dose $>2$ Gy, esta deve ser registrada em prontuário, e o paciente, notificado, para posterior seguimento. ${ }^{23,28}$

Um cardiologista intervencionista experiente, que realize um grande volume de cateterismos, tem uma exposição equivalente aproximada de $5 \mathrm{mSv}$ por ano e risco acumulado pela atividade laboral de $1 \mathrm{em}$ $100 .{ }^{57,58} \mathrm{O}$ risco é cumulativo para o paciente e o médico; isso significa que, quanto maior o número de procedimentos realizados, aumentam também a dose e os riscos. De acordo com estimativas de risco atuais, se 100 indivíduos são expostos a 100 mSv, 42 terão um câncer espontâneo (independentemente da exposição à radiação) e 1 terá um câncer induzido pela radiação (com incerteza de 1 em 30 a 1 em 300). O risco é três a quatro vezes maior em crianças e $50 \%$ menor em populações com mais de 80 anos, comparado com um grupo de pacientes com 50 anos de idade, e 38\% maior em mulheres que em homens. ${ }^{29,57}$

No Brasil, os limites de doses para o IOE estão estabelecidos pela portaria $453 .{ }^{16}$ Estabeleceu-se que a dose efetiva média anual não deve exceder os 20 mSv em qualquer período de 5 anos consecutivos, não podendo exceder $50 \mathrm{mSv}$ em nenhum ano. A dose equivalente anual não deve exceder 500 mSv para extremidades e $150 \mathrm{mSv}$ para o cristalino.

Limites de doses não se aplicam a pacientes, porque o objetivo é o diagnóstico ou o tratamento. Aplicam-se os níveis de referência, aos quais se podem comparar as doses de radiação entre grupos, para verificar se os valores recomendados para determinada prática estão sendo respeitados. Se o resultado das doses é maior, é necessária a revisão do equipamento e da forma de trabalho; se são obtidos níveis mais baixos, é necessária a revisão da qualidade das imagens, que podem estar aquém da desejada.

\section{Recomendações}

Em geral, deve-se insistir na educação e na capacitação dos profissionais e da sociedade. É necessário que o ensino da proteção radiológica seja introduzido já no nível da graduação nas faculdades de Medicina. Os hospitais deveriam disponibilizar cursos sistemáticos para proteção radiológica. Investir em segurança é investir na saúde.

Um dos objetivos da proteção radiológica é evitar o uso desnecessário da radiação ionizante, justificando adequadamente cada procedimento (princípio da justificação). ${ }^{59}$ Esse princípio estabelece que a exposição 
à radiação deve produzir um benefício suficiente para compensar o dano que a radiação possa causar. ${ }^{6-13}$ Os procedimentos devem ser feitos com a mínima dose de radiação.

Deve-se aprimorar a formação dos profissionais envolvidos em cardiologia intervencionista na área de proteção radiológica ${ }^{19-23}$; além disso, os equipamentos emissores de radiação ionizante devem estar submetidos a programas de garantia da qualidade que possibilitem reduzir a exposição às radiações ionizantes a níveis tão baixo quanto razoavelmente exequíveis (princípio ALARA, acrônimo do inglês As Low As Reasonably Achievable), ${ }^{9-13}$ minimizando os riscos para o paciente e a equipe médica.

O princípio da otimização preconiza um balanço entre risco e benefício. Potenciais riscos da radiação são admitidos, desde que haja uma adequada gestão da dose para todos os procedimentos de imagenologia. ${ }^{59-61}$
Existem diversas publicações internacionais e algumas nacionais sobre as recomendações e metodologias para o gerenciamento da dose, a proteção do paciente e do staff, e tutoriais em física das radiações, as quais se fazem fundamentais para a prática da segurança e

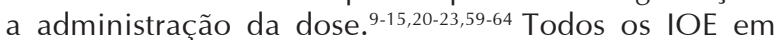
procedimentos que utilizem fluoroscopia deveriam se familiarizar com esses documentos e com outras literaturas relevantes nessa área.

Em geral, a adoção de práticas que protegem o paciente também minimiza os riscos da equipe médica. O médico operador é responsável pelo procedimento intervencionista e deve ter consciência dos riscos das radiações ionizantes. Os quadros 1 e 2, publicados pela IAEA em 2010,62,63 apresentam recomendações sobre proteção radiológica no laboratório de Cardiologia Intervencionista.

Todos os indivíduos que participam do procedimento intervencionista devem dispor dos elementos de

\section{QUADRO 1}

\section{Dez recomendações para proteção de pacientes em fluoroscopia ${ }^{62}$}

1. Maximizar, na medida do possível, a distância entre o tubo de raios $\mathrm{X}$ e o paciente

2. Minimizar a distância entre o paciente e 0 intensificador de imagem

3. Minimizar o tempo de fluoroscopia. Arquivar os dados de tempo de fluoroscopia para cada paciente

4. Usar fluoroscopia pulsada com a menor quantidade de pulsos possível para obter imagens com qualidade aceitável

5. Evitar expor a mesma região da pele nas diferentes projeções. Mude o ponto de entrada do feixe, girando o tubo em torno do paciente

6. Pacientes com maior espessura tecidual requerem aumento da ESD

7. Projeções oblíquas também aumentam a ESD. Note que 0 aumento da ESD eleva a probabilidade de dano na pele

8. Evitar 0 uso da ampliação. Diminuindo o campo visual para um fator 2, a dose é aumentada em um fator 4

9. Minimizar o número de quadros e o número de séries a um nível clinicamente aceitável. Evitar usar o modo de aquisição se é possível usar a fluoroscopia 10. Usar colimação. Colimar o feixe de raios $X$ na área de interesse

EDS: sigla do termo em inglês entrance surface dose, dose na superfície de entrada.

QUADRO 2

Dez recomendações para a proteção da equipe de trabalho em fluoroscopia ${ }^{63}$

1. Use dispositivos de proteção

2. Faça bom uso dos princípios tempo-distância-blindagem: minimize o tempo, maximize a distância o quanto seja possível clinicamente, e use a blindagem

3. Use os biombos de teto, as blindagens laterais e as cortinas plumbíferas abaixo da mesa ou o saiote

4. Mantenha suas mãos fora do feixe primário de raios $\mathrm{X}$ em todo momento

5. Somente 1 a $5 \%$ da radiação que incide na entrada do paciente vai para o detector de imagem

6. Mantenha o tubo de raios $X$ abaixo da mesa, e não sobre ela

7. Use seu dosímetro pessoal

8. Atualize periodicamente seus conhecimentos em proteção radiológica

9. Exponha suas dúvidas sobre radioproteção aos especialistas em proteção radiológica (especialistas em física médica)

10. Lembre:

- 0 controle de qualidade dos equipamentos de fluoroscopia permite trabalhar de forma segura

- Conheça seu equipamento. Utilizar as características do equipamento de maneira apropriada ajuda a reduzir a dose ao paciente e a equipe

- Use bombas injetoras de meio de contraste automáticas 
proteção pessoal. Há um avental que é especialmente desenhado para a prática, podendo ser tubular e com cinto para a distribuição do peso, e um conjunto (jaqueta e saia), com uma proteção equivalente a $0,5 \mathrm{mmPb}$, que atenua aproximadamente $95 \%$ da radiação espalhada para 70 kV. Para a proteção do cristalino são necessários óculos com proteção equivalente a $0,25 \mathrm{mmPb}$ e blindagem lateral. Adicionalmente, deve se incorporar o protetor de tireoide, com uma proteção de $0,35 \mathrm{mmPb}^{15}$

As roupas de proteção pessoal precisam de um cuidado diário, que involve a maneira adequada de armazenamento, limpeza e inspeção periódica em busca de danos nos trajes de proteção.

Outro sistema de blindagem para a proteção do operador são os elementos externos de proteção, como o saiote e o biombo de teto, os quais também são fornecidos pelos fabricantes dos angiógrafos. O biombo móvel proporciona proteção para a equipe médica que fica dentro da sala de procedimentos. Esses elementos oferecem uma significativa proteção contra a radiação espalhada, embora existam operadores que os considerem de difícil manipulação. O saiote proporciona proteção eficiente da parte inferior do tronco do operador, em especial para as gônadas, e seu uso é habitual em todos os procedimentos intervencionistas.

O biombo de teto transparente oferece uma proteção significativa para a parte superior do tronco do operador em especial a cabeça, a tireoide e o cristalino. ${ }^{23}$ Ao posicionar o biombo de teto, é necessário considerar a origem da radiação espalhada provocada pelo paciente e, em seguida, colocar a proteção para atenuar essa radiação. Um biombo de teto de $1 \mathrm{mmPb}$ e os óculos com uma proteção equivalente a $0,5 \mathrm{mmPb}$ podem chegar a diminuir a radiação espalhada que atinge $o$ cristalino em um fator 0,015 e 0,03 respectivamente, ${ }^{39}$ ou seja, somente 2 a 3\% (98 a 97\% de atenuação) da radiação espalhada seria transmitida ao cristalino. Esses fatores podem variar de acordo a dinâmica do procedimento e, principalmente, pela modificação da qualidade da radiação dispersa.

Outros estudos mostram que os óculos com proteção de $0,75 \mathrm{mmPb}$ podem atenuar entre 30 a $88 \%$ da radiação espalhada devido ao retroespalhamento da cabeça e ao ângulo de incidência da radiação. A pesquisa de McVey et al. ${ }^{64}$ confirmou que o retroespalhamento provocado pela cabeça do operador é a principal limitação para a redução da dose fornecida pelos óculos de proteção. Assim, o uso do biombo de teto é fortemente recomendado.

O uso adequado dos elementos de proteção radiológica pode reduzir significativamente a exposição à radiação da equipe de Cardiologia Intervencionista evitando, por exemplo, a opacidade do cristalino. Por outro lado, a variabilidade no poder de atenuação dos elementos de proteção radiológica e o fato de que a forma de realizar um determinado procedimento é operador-dependente fazem com que as principais fontes de proteção sejam a consciência e conhecimento que o operador tem do risco radiológico - muitas vezes subvalorizado.

\section{AGRADECIMIENTOS}

Aos doutores Carlos Ubeda, da Universidade de Tarapacá do Chile, e Alejandro Nader, oficial técnico de proteção contra as radiações em medicina da International Atomic Energy Agency, pela contribuição intelectual e técnica. O autor F.L. agradece a pós-graduação do Centro de Desenvolvimento da Tecnologia Nuclear (CDTN) e a Comissão Nacional de Energia Nuclear (CNEN) pela bolsa de doutorado.

\section{CONFLITO DE INTERESSES}

Não há.

\section{FONTE DE FINANCIAMENTO}

Não há.

\section{REFERÊNCIAS}

1. National Council on Radiation Protection and Measurements. lonizing radiation exposure of the population of the United States. Bethesda: NCPR; 2009. (NCRP Report n. 160).

2. United Nations Scientific Committee on Effects of Atomic Radiations. Source and effects of ionizing radiation. UNSCEAR 2008: report to the General Assembly with Scientific Annexes. New York: United Nations; 2011. v.2.

3. Food and Drug Administration. Important Information for Physicians and Other Health Care Professionals. Avoidance of serious x-ray induced skin injuries to patients during fluoroscopically guided procedures [Internet]. Rockville; 1994 [cited 2013 July 4]. Available from: http://www.fda.gov/downloads/ Radiation-EmittingProducts/RadiationEmittingProductsand Procedures/Medicallmaging/MedicalX-Rays/ucm116677.pdf

4. Limacher MC, Douglas PS, Germano G, Laskey WK, Lindsay BD, McKetty MH, et al.; American College of Cardiology. ACC Expert Consensus Document. Radiation safety in the practice of cardiology. J Am Coll Cardiol. 1998;31(4):892-913.

5. European Commission. Council Directive 97/43EURATOM of 30 June 1997. On health protection of individuals against the dangers of ionizing radiation in relation to medical exposure, and repealing Directive 84/466/Euratom [Internet]. [cited 2013 July 4]. Available from: http://ec.europa.eu/energy/nuclear/ radioprotection/doc/legislation/9743_en.pdf

6. European Commission. Guidelines on education and training in radiation protection for medical exposures. Radiation Protection 116 [Internet]. Luxemburgo; 2000 [cited 2013 July 4]. Available from: http://ec.europa.eu/energy/nuclear/ radiation_protection/doc/publication/116.pdf

7. Gov UK. Department of Health. Ionising radiation (Medical Exposures): regulations 2000 (ISMER) [Internet]. London; 2012 [cited 2013 July 2]. Available from: https://www.gov. uk/government/publications/the-ionising-radiation-medicalexposure-regulations-2000 
8. Partridge J. Education in heart radiation in the cardiac catheter laboratory. Heart. 2005;91(12):1615-20.

9. Organismo Internacional de Energía Atómica. Protección radiológica y seguridad de las fuentes de radiación: normas básicas internacionales de seguridad. Edición provisional. Requisitos de seguridad generales. Viena: OIEA; 2011.

10. International Commission on Radiological Protection. The 1990 Recommendations. ICRP Publication 60. Ann ICRP. $1991 ; 21(1-3)$.

11. International Commission on Radiological Protection. The 2007 Recommendations. ICRP Publication 103. Ann ICRP. $2007 ; 37(2-4)$.

12. International Commission on Radiological Protection. Radiological protection in medicine. ICRP Publication 105. Ann ICRP. 2007;37(6)

13. Chambers CE, Fetterly KA, Holzer R, Lin PJ, Blankenship JC, Balter S, et al. Radiation safety program for the cardiac catheterization laboratory. Catheter Cardiovasc Interv. 2011; 77(4):546-56.

14. Chambers CE. Radiation dose: it is more than just "time". Catheter Cardiovasc Interv. 2011;78(1):143-4.

15. International Commission on Radiological Protection. Avoidance of radiation injuries from medical interventional procedures. ICRP Publication 85. Ann ICRP. 2000;30(2).

16. Brasil. Ministério da Saúde. Portaria n. 453, de 1 de junho de 1998. Dispões sobre as Diretrizes de Proteção Radiológica em Radiodiagnóstico Médico e Odontológico. Diário Oficial da União, Brasília, 2 jun. 1998.

17. Brasil. Ministério do Trabalho e Emprego. NR 32 - Segurança e Saúde no Trabalho em Serviços de Saúde [Internet]. Brasília; 2011 [citado 2013 jun. 10]. Disponível em: http://portal.mte. gov.br/data/files/8A7C816A350AC8820135161931EE29A3/NR32 (atualizada 2011).pdf

18. Vano E, Ubeda C, Miranda P, Leyton F, Durán A, Nader A. Radiation protection in pediatric interventional cardiology: an IAEA PILOT program in Latin America. Health Phys. 2011;101(3):233-7.

19. International Atomic Energy Agency. Cardiology Training Events. Regional training courses on radiation protection in cardiology organized under TC support [Internet]. [cited 2013 July 2]. Available from: https://rpop.iaea.org/RPOP/RPoP/Content-es/ AdditionalResources/Training/2_TrainingEvents/Cardiology.htm

20. Hirshfeld JW, Balter S, Brinker JA, Kern MJ, Klein LW, Lindsay $\mathrm{BD}$, et al. ACCF/AHA/HRS/SCAI clinical competence statement on physician knowledge to optimize patient safety and image quality in fluoroscopically guided invasive cardiovascular procedures: a report of the American College of Cardiology Foundation/American Heart Association/American College of Physicians Task Force on Clinical Competence and Training. Circulation. 2005;111(4):511-32.

21. Bashore TM, Bates ER, Berger PB, Clark DA, Cusma JT, Dehmer GJ, et al. American College of Cardiology/ Society for Cardiac Angiography and Interventions Clinical Expert Consensus Document on cardiac catheterization laboratory standards. A report of the American College of Cardiology Task Force on Clinical Expert Consensus Documents. J Am Coll Cardiol. 2001;37(8):2170-214.

22. International Commission on Radiological Protection. Education and training in radiological protection for diagnostic and interventional procedures. ICRP Publication 113. Ann ICRP. 2009;39(5).

23. National Council on Radiation Protection and Measurements. Radiation dose management for fluoroscopically-guided interventional medical procedures. Bethesda; 2011. (NCRP Report, n. 168).
Proteção Radiológica na Cardiologia Intervencionista

24. Wiesinger B, Stütz A, Schmehl J, Claussen CD, Wiskirchen J. Comparison of digital flat-panel detector and conventional angiography machines: evaluation of stent detection rates, visibility scores, and dose-area products. AJR Am J Roentgenol. 2012;198(4):946-54

25. Chida K, Inaba Y, Saito H, Ishibashi T, Takahashi S, Kohzuki M, et al. Radiation dose of interventional radiology system using a flat-panel detector. AJR Am J Roentgenol. 2009;193(6):1680-5.

26. Medeiros RF, Sarmento-Leite R, Cardoso CO, Quadros AS, Risso E, Ficher L, et al. Exposição à radiação ionizante na Sala de Hemodinâmica. Rev Bras Cardiol Invasiva. 2010;18(3):316-20.

27. Vargas FG, Silva BS, Cardoso CO, Leguisamo N, Moraes CAR, Moraes CV, et al. Impacto do peso corporal dos pacientes na exposição radiológica durante procedimentos cardiológicos invasivos. Rev Bras Cardiol Invasiva.2012;20(1):63-8.

28. International Atomic Energy Agency. Establishing guidance levels in $X$ ray guided medical interventional procedures: a pilot study. Vienna; 2009. (Safety Reports Series, n. 59).

29. Picano E, Vano E. The radiation issue in cardiology: the time for action is now. Cardiovasc Ultrasound. 2011;9:35.

30. Cascón A. Riesgos asociados con las radiaciones ionizantes. Rev Argent Cardiol. 2009;77(2):123-8.

31. United Nations Scientific Committee on the Effects of Atomic Radiation. Sources and effects of ionizing radiation. UNSCEAR 2000: Report to the General Assembly with scientific annexes. New York: United Nations; 2000. v.1

32. National Council on Radiation Protection and Measurement. Evaluation of the linear-nonthreshold dose-response model for ionizing radiation. Bethesda; 2002. (NCRP Report, n. 136).

33. National Research Council. Committee to Assess Health Risks from Exposure to Low Levels of Ionizing Radiation. Health risks from exposure to low levels of ionizing radiation: BEIR VII Phase 2. Washington: National Academies; 2006.

34. International Commission on Radiological Protection. Low-dose extrapolation of radiation-related cancer risk. ICRP Publication 99. Ann ICRP. 2005;35(4).

35. Koenig TR, Wolff D, Mettler FA, Wagner LK. Skin injuries from fluoroscopically guided procedures: part 1, characteristics of radiation injury. AJR Am J Roentgenol. 2001;177(1):3-11.

36. Koenig TR, Mettler FA, Wagner LK. Skin injuries from fluoroscopically guided procedures: part 2, review of 73 cases and recommendations for minimizing dose delivered to patient. AJR Am J Roentgenol. 2001;177(1):13-20.

37. Iyer PS. Acute radiation injury caused by faulty x-ray fluoroscopy during cardiac procedures. Health Phys. 1976;31(4):385-7.

38. International Atomic Energy Agency; European Commission; Pan American Health Organization; World Health Organization. Radiological protection of patients in diagnostic and interventional radiology, nuclear medicine and radiotherapy. In: Proccedings of an International Conference Held in Málaga, Spain, 26-30 March 2001. Vienna: IAEA; 2001.

39. Vañó E, Gonzalez L, Fernández JM, Haskal ZJ. Radiation exposure to the lens in interventional suites: caution is warranted. Radiology. 248(3):945-53.

40. Vañó E, González L, Guibelalde E, Fernández JM, Ten J. Radiation exposure to medical staff in interventional and cardiac radiology. Br J Radiol. 1998;71(849):954-60.

41. Chodick G, Bekiroglu N, Hauptmann M, Alexander BH, Freedman DM, Doody MM, et al. Risk of cataract after exposure to low doses of ionizing radiation: a 20-year prospective cohort study among US radiologic technologists. Am J Epidemiol. 2008; 168(6):620-31.

42. Vano E, Kleiman NJ, Duran A, Romano-Miller M, Rehani MM. Radiation-associated lens opacities in catheterization personnel: results of a survey and direct assessments. J Vasc Interv Radiol. 2013;24(2):197-204. 
43. International Atomic Energy Agency. Radiation Protection of Pacients. IAEA Cataract study. AEA activity on Retrospective Evaluation of Lens Injuri and Dose (RELID) [Internet]. [cited 2013 July 2]. Available from: https://rpop.iaea.org/rpop/rpop/ content/news/relid-cataract-study.htm

44. Russo GL, Tedesco I, Russo M, Cioppa A, Andreassi MG, Picano E. Cellular adaptive response to chronic radiation exposure in interventional cardiologists. Eur Heart J. 2012;33(3):408-14.

45. Almeida Junior AT, Alonso TC, Nogueira MS, Silva TA. Dose levels in medical staff at hemodynamics services in Minas Gerais State, Brazil:preliminary results. Radiat Meas. 2008; 43(2):917-20.

46. Fetterly KA, Magnuson DJ, Tannahill GM, Hindal MD, Mathew $\checkmark$. Effective use of radiation shields to minimize operator dose during invasive cardiology procedures. JACC Cardiovasc Interv. 2011;4(10):1133-9.

47. International Commission on Radiological Protection. Statement on Tissue Reactions. Approved by the Commission on April 21, 2011 [Internet]. [cited 2013 July 2]. Available from: http://www.icrp.org/docs/icrp\%20statement\%20on\%20tissue\%20 reactions.pdf

48. International Atomic Energy Agency. Colección de normas de seguridad № RS-G-1.5. Protección radiológica relacionada con la exposición médica a la radiación ionizante. Viena: IAEA: 2010.

49. Ubeda C, Vano E, Miranda P, Leyton F. Pilot program on patient dosimetry in pediatric interventional cardiology in Chile. Med Phys. 2012;39(5):2424-30.

50. Suzuki S, Furui S, Issiki T, Kozuma K, Koyama Y, Yamamoto $\mathrm{H}$, et al. Patients skin dose during percutaneous intervention for chronic total occlusion. Catheter Cardiovasc Interv. 2008; 71(2):160-4.

51. Panuccio G, Greenberg RK, Wunderle K, Mastracci TM, Eagleton MG, Davros W. Comparison of indirect radiation dose estimates with directly measured radiation dose for patients and operators during complex endovascular procedures. J Vasc Surg. 2011;53(4):885-94.

52. Signorotto $P$, Del Vecchio A, Montorfano $M$, Maisano $F$, Giagnorio M, Bellanca R, et al. Dosimetric data and radiation risk analysis for new procedures in interventional cardiology. Radiat Prot Dosimetry. 2010;142(2-4):201-8.

53. Gerber TC, Carr JJ, Arai AE, Dixon RL, Ferrari VA, Gomes $\mathrm{AS}$, et al. lonizing radiation in cardiac imaging: a science advisory from the American Heart Association Committee on Cardiac Imaging of the Council on Clinical Cardiology and
Committee on Cardiovascular Imaging and Intervention of the Council on Cardiovascular Radiology and Intervention. Circulation. 2009;119(7):1056-65

54. Mettler FA Jr, Huda W, Yoshizumi TT, Mahesh M. Effective doses in radiology and diagnostic nuclear medicine: a catalog. Radiology. 2008;248(1):254-63.

55. Azevedo EM, Gomes HB, Yordi LM, Moura MRS, Laguna A, Fischer LS, et al. Impacto das lesões complexas na exposição radiológica durante intervenção coronária percutânea. Rev Bra Cardiol Invasiva. 2013;21(1):49-53.

56. Mattos El, Cardoso CO, Moraes CV, Teixeira JVS, Azmus AD, Fischer LS, et al. Exposição radiológica em procedimentos coronários realizados pelas vias radial e femoral. Rev Bras Cardiol Invasiva. 2013;21(1):54-9.

57. Venneri L, Rossi F, Botto N, Andreassi MG, Salcone N, Emad A, et al. Cancer risk from professional exposure in staff working in cardiac catheterization laboratory: insights from the National Research Councils Biological Effects of lonizing Radiation VII Report. Am Heart J. 2009;157(1): 118-24.

58. International Atomic Energy Agency. Justification of Medical Exposure in Diagnostic Imaging. In: Proceedings of an International Workshop; Brussels, 2-4 Sept 2009 [Internet]. Vienna: IAEA; 2009 [cited 2013 July 2]. Available from: http://wwwpub.iaea.org/MTCD/Publications/PDF/Pub1532_web.pdf

59. Balter S, Moses J. Managing patient dose in interventional cardiology. Catheter Cardiovasc Interv. 2007;70(2):244-9.

60. Stecker MS, Balter S, Towbin RB, Miller DL, Vano E, Bartal $\mathrm{G}$, et al. Guidelines for patient radiation management. J Vasc Inter Radiol. 2009;20(7 Suppl:S263-73.

61. Canevaro L. Aspectos físicos e técnicos da radiologia intervencionista. Rev Bras Física Méd. 2009;3(1):101-15.

62. International Atomic Energy Agency. 10 Recomendaciones para protección de pacientes en fluoroscopía [Internet]. [citado 2013 Jul. 2]. Disponible en: http://rpop.iaea.org/RPOP/RPoP/ Content/Documents/Whitepapers/poster-patient-radiationprotection-es.pdf

63. International Atomic Energy Agency. 10 Recomendaciones para la protección del staff en fluoroscopía [Internet]. [citado 2013 Jul. 2]. Disponible en: http://rpop.iaea.org/RPOP/ RPoP/Content/Documents/Whitepapers/poster-staff-radiationprotection-es.pdf

64. McVey S, Sandison A, Sutton DG. An assessment of lead eyewear in interventional radiology. J Radiol Prot. 2013;33(3): $647-59$. 